\title{
The association of quality of life and personality characteristics with adolescent metabolic syndrome: a cohort study
}

\author{
Xiaohua Liang ${ }^{1^{*}} \mathbb{D}$, Peng Zhang ${ }^{2}$, Shunqing Luo ${ }^{3}$, Guifang Zhang ${ }^{4}$, Xian Tang ${ }^{1}$ and Lingjuan Liu ${ }^{1}$
}

\begin{abstract}
Background: An increased prevalence of adolescent metabolic syndrome (MS) is associated with adulthood cardiovascular diseases. This study aimed to explore the potential relationship of quality of life (QoL) and personality traits with adolescent MS.
\end{abstract}

Methods: A total of 1961 participants from Chongqing with an average age of 11.68 years old from a cohort study established in 2014 and followed up through 2019 were included. QoL information, Eysenck's personality questionnaire and MS components were collected.

Results: A higher QoL domain score of physical activity ability (PAA) was a protective factor for both MS and MS score (all $P<0.01$ ), which was mainly negatively correlated with the MS components of central obesity, diastolic blood pressure (DBP) and triglyceride levels, as well as positively correlated with high density lipoprotein cholesterol (HDLC) level. The total QoL score was negatively correlated with triglyceride levels and positively correlated with DBP (all $P<0.01)$. High extraversion personality score was a protective factor against adolescent MS $(P=0.04)$ and MS score $(P<0.05)$, which were mainly negatively correlated with the MS components of waist circumference, systolic blood pressure and TGs, and positively correlated with HDL-C (all $P \leq 0.01$ ).

Conclusions: QoL score and extraversion personality score were independent protective factors against both MS prevalence and MS score, suggesting that community intervention to improve the QoL and psychological health of children are essential.

Keywords: Quality of life, Personality traits, Adolescent, Metabolic syndrome, Cohort study

\section{Introduction}

The increased prevalence of adolescent metabolic syndrome (MS) and MS component severity scores are independent predictors of adulthood cardiovascular

\footnotetext{
*Correspondence: xiaohualiang@hospital.cqmu.edu.cn

${ }^{1}$ Clinical Epidemiology and Biostatistics Department, Children's Hospital of Chongqing Medical University, Ministry of Education Key Laboratory of Child Development and Disorders, National Clinical Research Center for Child Health and Disorders, Key Laboratory of Pediatrics in Chongqing, China International Science and Technology Cooperation Center of Child Development and Critical Disorders, 136 2nd Street, Yuzhong District, Chongqing 400016, China

Full list of author information is available at the end of the article
}

diseases (CVDs) [1, 2]. MS components in adolescents include central obesity, elevated triglycerides (TGs), reduced high density lipoprotein cholesterol (HDL$\mathrm{C})$, elevated blood pressure and impaired fasting glucose. The prevalence of MS among adolescents ranged from 3.5 to $11.2 \%$ according to different regions and different diagnostic criteria [3, 4]. Current studies [5, 6] have found that shared genetic and environmental factors, family history of CVD and obesity, maternal gestational diabetes [7], low birth weight, early adiposity, social economic status (SES) [8], short duration of sleep [9], excessive screen time, dietary factors, low physical activity, and tobacco smoke exposure 
were potential risk factors for adolescent MS or MS components. The literature has consistently demonstrated evidence for the association of physical health risks and socioeconomic status with adolescent MS or its components; however, the association of healthrelated quality of life (QoL) and personality traits with adolescent MS should also gain the attention of researchers.

Limited studies [10] have illustrated the inverse relationship of QoL with MS or MS components mainly in adults, and the conclusions have been controversial. However, the majority of studies have shown that the association existed only in women [11] or existed only in subjects with depression [12]. Previous studies have revealed that the QoL domain scores of physical health [13] and social relationships [14], but not those of the mental health and perceived stress domains, were correlated with MS. Moreover, the impact factors of QoL were age, obesity, puberty development stage, SES, physical activity $[15,16]$ and unhealthy dietary behaviour [17], which were also correlated with MS. In addition, one community obesity intervention study [18] revealed that the control of obesity was associated with QoL improvement. Considered together, these studies suggest that QoL could have significant effects on MS, but evidence in children and adolescents has been scarce. Therefore, it is urgent to explore the association of QoL with adolescent MS and reveal the QoL domains that have salient effects.

Personality traits can impact MS and its components, although few studies have investigated this association. One study showed that children with obesity might experience several psychosocial problems [19]. A study found that extraverted personality is positively correlated with TGs, fasting blood-glucose (FBG) and MS scores in adults [20]. However, to our knowledge, there have been no studies exploring the correlations between personality traits and MS in adolescents, and personality traits tend to be stable over time and consistent across situations [21]. Therefore, personality traits could impact the prevalence of MS among children and adolescents, which must be illustrated.

In this study, we investigated whether QoL scores were inversely correlated with adolescent MS and MS components, which domains have salient effects, and whether the association was independent of age, sex, region and other variables. We investigated whether personality traits (extraversion, neuroticism, psychoticism) were associated with MS and MS components, and whether the association was independent of the influence of sex, age and other factors. A hypothesized framework figure is drawn in Additional file 1: Figure S1.

\section{Methods}

\section{Subjects}

Two stage stratified cluster sampling was used to include participants from two counties in Chongqing that represent urban and rural areas; then, two regions per county were randomly selected, and 3067 children (including 2808 children who entered the cohort in 2014, and 130 and 129 children who transferred to the target schools in 2015 and 2019, respectively) were ultimately informed and included if they met the inclusion criteria. Participants who met all of the following criteria were recruited: (1) aged between 6 and 9 years old in 2014; (2) resided in the target region for more than 6 months; (3) did not have serious diseases (e.g., nephropathy, cardiovascular disease or cancer); and (4) had consent provided by the parents and children for participation. The sample size was calculated with the following parameters: $\alpha$ level of 0.05 , power of $90 \%$, prevalence of MS components of $15 \%$ and prevalence in the population of $10 \%$, using the formula $n=p(1-p)\left(\frac{z_{1-\alpha / 2}+z_{1}-\beta}{p-p_{0}}\right)^{2}$. Assuming an attrition rate of 20\%, 1859 participants were needed, and 1961 subjects were ultimately included in this study (as shown in Fig. 1). The general characteristics of the included and excluded samples are described in Additional file 2: Table S1. At baseline, all of the participants completed the SES and family health history questionnaires and were recruited mainly from grade one and grade two based on primary public school screening of children whose families were interested in health research. The questionnaires were administered and collected by the teachers. The Institutional Review Board at the Children's Hospital of Chongqing Medical University provided approval for the study with an ethics approval number (2019-86). Informed consent was provided by all of the subjects and parents/guardians.

\section{Demographic variables}

Demographic information, SES and prenatal variables were collected. The validity and reliability of the demographic questionnaire were checked and are described in detail in a previous publication [22]. The demographic questionnaire was completed by the parents or guardians of the children after standard training by the research group, and detailed instructions regarding the questionnaire were given to the parents or guardians.

\section{Physical examination}

Anthropometric measurements were conducted both in 2014 and in 2019 by well-trained paediatric nurses, and the protocol for these measurements was described in a previous publication [22]. Waist circumference (WC) 


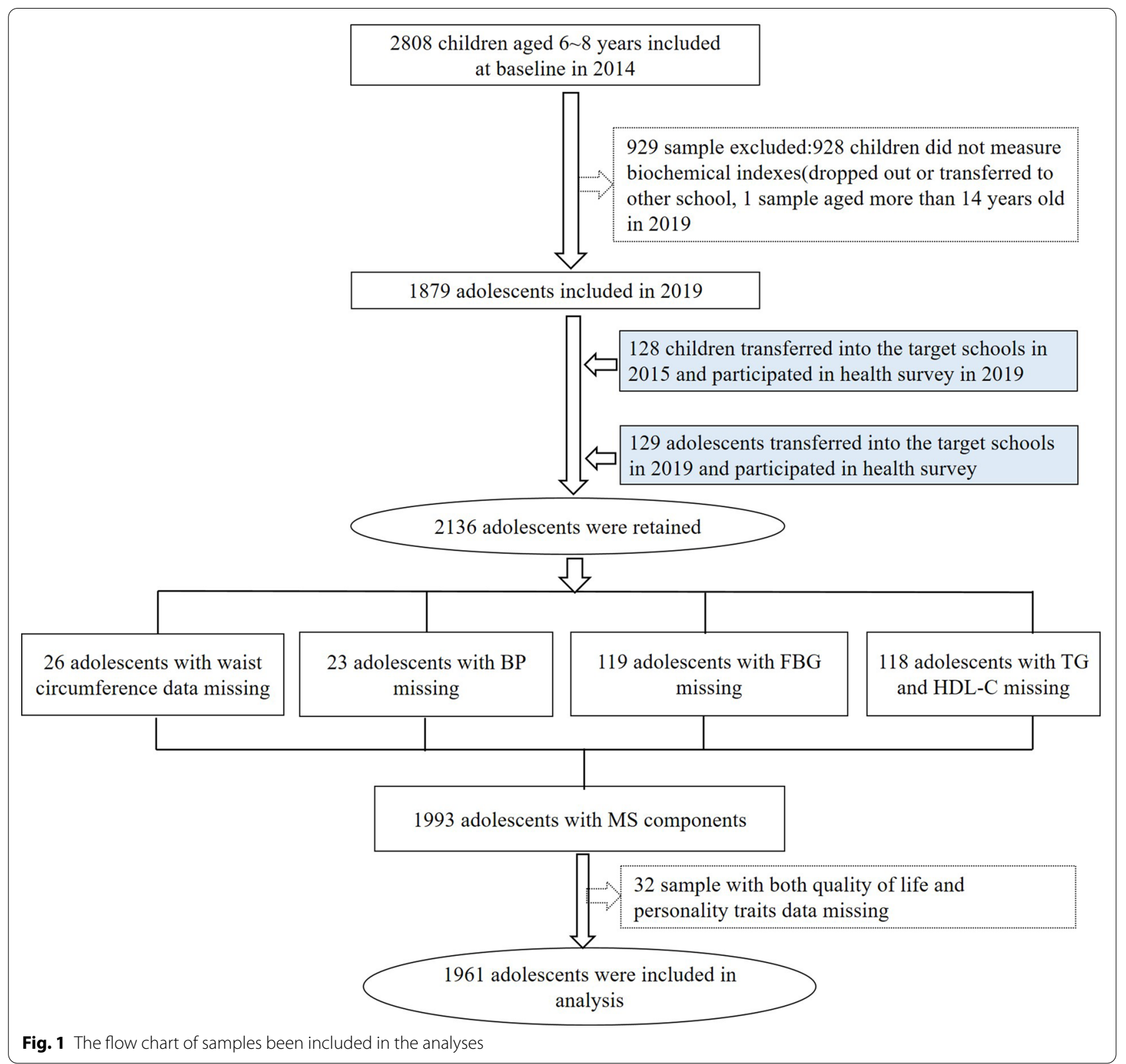

was used as an alternative measure of central adiposity. Hip circumference was measured twice horizontally at the level of the pubic symphysis in the front and the gluteus maximus in the back, with the participant standing upright and with his or her legs together and placing the arms naturally at the sides; the mean value was used.

Blood pressure (BP) was measured on three separate occasions with an OMRON arm-type electronic sphygmomanometer (HEM7051) using an appropriately sized BP cuff placed on the subject's right arm and with the subject in a seated position, as described in detail in a previous publication [22].

\section{Biochemical indexes}

The biochemical markers FBG, HDL-C, TG, low density lipoprotein cholesterol (LDL), and total cholesterol (TC) were measured in 2014 and 2019. Venous blood $(3 \mathrm{~mL})$ was drawn from each subject in the morning after at least $12 \mathrm{~h}$ of fasting and $24 \mathrm{~h}$ of abstaining from high-fat and spicy foods. The biochemical markers were measured within $2 \mathrm{~h}$ after venous blood was drawn using the protocol introduced in detail in our previous publication [22]. 


\section{Measurement of QoL and Eysenck's Personality Questionnaire (EPQ)}

The QoL questionnaire for adolescents consists of 49 items, including 4 factors (psychosocial function, physical and mental health, living environment and QoL satisfaction) and 13 dimensions, including selfsatisfaction, relationship of teacher and pupil, physical feeling, companionship, parenthood, physical activity ability (PAA), learning ability and attitude, self-esteem, negative emotion, attitude towards completing homework, opportunity for activity, living convenience and others (picky-eating and surroundings), as detailed in Additional file 3: Figure S2. The order of presentation of the 49 items was randomized. Children rated the statements on a 4-point scale, and the direction of response (positive or negative) varied item by item to limit response bias. Individual item values were recoded prior to analysis such that the direction was consistent. Responses were totalled and normalized according to the age-, sex- and region-specific norms of China into a score $\mathrm{T}$ (range $0-100$ ), using the function $\mathrm{T}=50+(\mathrm{X}-\mathrm{M}) / \mathrm{SD} \times 10$, with higher scores suggesting a better QoL status [23].

The Chinese version of the Eysenck personality questionnaire [24] consisted of 88 items scored on a 2-point scale (for positive items $\mathrm{NO}=0$ and $\mathrm{YES}=1$ ), including 4 domains: extraversion (E) (25 items), neuroticism (N) (23 items), psychoticism (P) (18 items), and lie scales (L) (22 items). High scorers on the $\mathrm{E}$ scale indicate sociable, exciting, pleasurable, carefree, and aggressive characteristics. A higher score on the $\mathrm{N}$ scale is more likely to indicate a worried and moody person who tends to suffer from emotional and psychosomatic disorders. The P scale was designed to measure behaviour patterns that could be considered schizoid or psychopathic in extreme cases. The $\mathrm{L}$ scale assesses response bias. Items in the $\mathrm{E}, \mathrm{N}, \mathrm{P}$ and $\mathrm{L}$ domains are totalled and normalized to the age- and sex-specific norms into a score ranging from 0 to 100 using the function $\mathrm{T}=50+(\mathrm{X}-\mathrm{M}) / \mathrm{SD} \times 10$. People were defined as middle type, tendency type and typical type based on $\mathrm{T}$ score $(\mathrm{E}$ and $\mathrm{N}$ ) ranges of $43.3-56.7,38.5-43.3$ or $56.7-61.5$, and $<38.5$ or $>61.5$, respectively. People were considered to have a psychotic personality if the $\mathrm{T}$ score of the $\mathrm{P}$ domain was $>56.7$. We considered the responses regarding personality traits invalid if the $\mathrm{T}$ score of the L domain was $>70$.

Fifty samples were required to complete the same questionnaire twice within a one-week interval to check the validity and reliability of the QoL and Eysenck's Personality Questionnaires before our formal survey. QoL and Eysenck's Personality Questionnaires were completed by the adolescents after a standard training.

\section{Diagnostic criteria}

MS in adolescents was defined by the presence of three or more of the following five components [5, 25]: (1) central obesity defined as $\geq 90$ th percentile for age and gender criteria in China [26]; (2) elevated systolic and/ or diastolic blood pressure $\geq 90$ th percentile for age, sex and height (according to the study by Jie Mi [27]); (3) hypertriglyceridaemia defined as $\mathrm{TG} \geq 1.24 \mathrm{mmol} / \mathrm{L}$; (4) low serum HDL-C defined as $\mathrm{HDL}-\mathrm{C} \leq 1.03 \mathrm{mmol} / \mathrm{L}$, and (5) impaired fasting glucose (IFG) defined as FBG $\geq 5.6 \mathrm{mmol} / \mathrm{L}$. Next, individual MS scores were calculated as the sum of the number of MS components present (range 0-5).

The definitions of size for gestational age were based on the global reference for foetal-weight and birthweight percentiles [28]: birth weight at or greater than the 90th percentile indicated large for gestational age (LGA), and birth weight less than the 10th percentile indicated small for gestational age (SGA), using the parameters of mean birthweight at 40.5 weeks of $3332.93 \mathrm{~g}$ and a variation coefficient of $14.36 \%$.

\section{Statistical analyses}

Differences in anthropometric measures, serum biochemical indexes, QoL and personality score among the three groups were assessed using ANOVA, and post hoc comparisons were performed using the Student-Newman-Keuls (SNK) test. Type I errors were already adjusted. We selected potential covariates if the difference was significant $(P<0.05)$, and compared the differences among MS component groups (No MS Components, 1-2 MS Components and MS). The $x^{2}$ test was used to test the difference in the component ratio of potential risk factors for MS components. A logistic regression model was performed using diagnosed MS components or MS as the dependent variables with QoL and personality traits, as independent variables, and adjusted for covariables. In addition, a generalized linear model (GLM) was used to analyse the correlation of QoL and personality trait scores with MS component levels and MS scores and adjusted covariables.

The data analysis was conducted using SAS software, version 9.4 (Copyright (C) 2020 SAS Institute Inc. Cary, NC, USA). A significant difference was defined by an $\alpha$ level of 0.05 .

\section{Results}

\section{General characteristics}

The general characteristics of the subjects are shown in Table 1. A total of 1961 samples were included, with a follow-up rate of $63.94 \%$ (1961/3067). The mean age was $11.68 \pm 0.60$ years old $(7.26 \pm 0.59$ years at baseline), and $52.01 \%(1020 / 1961)$ were male. The prevalence 
Table 1 General characteristics of adolescent QoL and personality traits with MS study

\begin{tabular}{|c|c|c|c|c|c|}
\hline \multirow[t]{2}{*}{ Variables } & \multirow[t]{2}{*}{ All } & \multicolumn{4}{|c|}{ Metabolic syndrome (MS) } \\
\hline & & No & $1 \sim 2$ MS Components & Yes & $P$ \\
\hline Sample size & 1961 & $1052(53.65 \%)$ & $817(41.66 \%)$ & $92(4.69 \%)$ & \\
\hline \multicolumn{6}{|l|}{ Region } \\
\hline Urban & $1467(74.81 \%)$ & $829(78.80 \%)$ & $576(70.50 \%)$ & $62(67.39 \%)$ & $<0.01$ \\
\hline Rural & $494(25.19 \%)$ & $223(21.20 \%)$ & $241(29.50 \%)$ & $30(32.61 \%)$ & \\
\hline Gender, male (n (\%)) & $1020(52.01 \%)$ & $554(52.66 \%)$ & $415(50.8 \%)$ & $51(55.43 \%)$ & 0.58 \\
\hline Age, y & $11.68 \pm 0.60$ & $11.65 \pm 0.60$ & $11.72 \pm 0.60$ & $11.75 \pm 0.60$ & 0.04 \\
\hline \multicolumn{6}{|c|}{ Anthropometric measures in 2014} \\
\hline Age, y & $7.26 \pm 0.59$ & $7.24 \pm 0.59$ & $7.28 \pm 0.58$ & $7.29 \pm 0.60$ & 0.33 \\
\hline Height, $\mathrm{cm}$ & $124.52 \pm 6.11$ & $123.67 \pm 5.86_{a}$ & $125.27 \pm 6.15_{b}$ & $127.57 \pm 6.72_{c}$ & $<0.01$ \\
\hline Weight, kg & $25.92 \pm 5.58$ & $24.27 \pm 4.13_{a}$ & $27.27 \pm 5.98 b$ & $32.65 \pm 7.61 c$ & $<0.01$ \\
\hline $\mathrm{BMI}, \mathrm{kg} / \mathrm{m} 2$ & $16.59 \pm 2.53$ & $15.79 \pm 1.82_{a}$ & $17.24 \pm 2.75_{b}$ & $19.84 \pm 3.09 c$ & $<0.01$ \\
\hline Waist circumference, $\mathrm{cm}$ & $55.69 \pm 7.09$ & $53.59 \pm 5.55 a$ & $57.40 \pm 7.48_{b}$ & $64.23 \pm 8.46_{c}$ & $<0.01$ \\
\hline $\mathrm{SBP}, \mathrm{mmHg}$ & $100.6 \pm 8.94$ & $98.85 \pm 8.36_{a}$ & $102.14 \pm 9.04_{b}$ & $106.70 \pm 9.33 c$ & $<0.01$ \\
\hline $\mathrm{DBP}, \mathrm{mmHg}$ & $62.68 \pm 7.76$ & $61.57 \pm 7.51_{\mathrm{a}}$ & $63.72 \pm 7.79_{b}$ & $66.08 \pm 8.04 c$ & $<0.01$ \\
\hline \multicolumn{6}{|c|}{ Anthropometric measures in 2019} \\
\hline Height, $\mathrm{cm}$ & $151.75 \pm 7.98$ & $150.22 \pm 7.89 a$ & $153.21 \pm 7.67_{b}$ & $156.33 \pm 7.59_{c}$ & $<0.01$ \\
\hline Weight, kg & $44.30 \pm 10.98$ & $39.63 \pm 7.69 a$ & $48.58 \pm 11.24_{b}$ & $59.62 \pm 11.30_{c}$ & $<0.01$ \\
\hline $\mathrm{BMI}, \mathrm{kg} / \mathrm{m}^{2}$ & $19.07 \pm 3.76$ & $17.45 \pm 2.50_{a}$ & $20.58 \pm 4.01_{b}$ & $24.22 \pm 3.32_{c}$ & $<0.01$ \\
\hline Waist circumference, $\mathrm{cm}$ & $65.83 \pm 10.03$ & $61.20 \pm 5.98 a$ & $70.06 \pm 10.77_{b}$ & $81.18 \pm 8.26_{c}$ & $<0.01$ \\
\hline Hip circumference, $\mathrm{cm}$ & $81.68 \pm 8.28$ & $78.45 \pm 6.49_{a}$ & $84.68 \pm 8.41_{b}$ & $92.05 \pm 6.58_{c}$ & $<0.01$ \\
\hline $\mathrm{SBP}, \mathrm{mmHg}$ & $105.71 \pm 9.49$ & $102.76 \pm 8.02_{a}$ & $108.29 \pm 9.62_{b}$ & $116.62 \pm 9.36_{c}$ & $<0.01$ \\
\hline $\mathrm{DBP}, \mathrm{mmHg}$ & $62.82 \pm 6.70$ & $61.40 \pm 5.87 a$ & $63.98 \pm 7.07_{b}$ & $68.72 \pm 7.05_{c}$ & $<0.01$ \\
\hline Puberty & $536(27.33 \%)$ & $267(25.38 \%)$ & $246(30.11 \%)$ & $23(25.00 \%)$ & 0.11 \\
\hline \multicolumn{6}{|c|}{ Serum biochemical indexes in 2014} \\
\hline $\mathrm{FBG}, \mathrm{mmol} / \mathrm{l}$ & $4.12 \pm 0.6$ & $4.07 \pm 0.59 \mathrm{a}$ & $4.17 \pm 0.58_{\mathrm{ab}}$ & $4.29 \pm 0.71_{b}$ & $<0.01$ \\
\hline $\mathrm{TC}, \mathrm{mmol} / \mathrm{l}$ & $3.51 \pm 0.72$ & $3.49 \pm 0.75$ & $3.54 \pm 0.68$ & $3.62 \pm 0.69$ & 0.25 \\
\hline $\mathrm{TG}, \mathrm{mmol} / \mathrm{l}$ & $0.91 \pm 0.53$ & $0.82 \pm 0.42_{a}$ & $0.99 \pm 0.58_{b}$ & $1.36 \pm 0.78_{c}$ & $<0.01$ \\
\hline $\mathrm{HDL}-\mathrm{C}, \mathrm{mmol} / \mathrm{l}$ & $1.26 \pm 0.27$ & $1.30 \pm 0.27 \mathrm{a}$ & $1.23 \pm 0.27 \mathrm{~b}$ & $1.10 \pm 0.22_{c}$ & $<0.01$ \\
\hline LDL-C, mmol/l & $1.73 \pm 0.56$ & $1.68 \pm 0.53_{a}$ & $1.78 \pm 0.59 \mathrm{a}$ & $1.99 \pm 0.63_{b}$ & $<0.01$ \\
\hline \multicolumn{6}{|c|}{ Serum biochemical indexes in 2019} \\
\hline $\mathrm{FBG}, \mathrm{mmol} / \mathrm{l}$ & $4.45 \pm 0.43$ & $4.45 \pm 0.38$ & $4.45 \pm 0.49$ & $4.50 \pm 0.51$ & 0.58 \\
\hline $\mathrm{TC}, \mathrm{mmol} / \mathrm{l}$ & $3.52 \pm 0.61$ & $3.53 \pm 0.57$ & $3.50 \pm 0.66$ & $3.47 \pm 0.62$ & 0.43 \\
\hline $\mathrm{TG}, \mathrm{mmol} / \mathrm{l}$ & $1.06 \pm 0.50$ & $0.83 \pm 0.20_{a}$ & $1.26 \pm 0.55_{b}$ & $1.85 \pm 0.84_{c}$ & $<0.01$ \\
\hline $\mathrm{HDL}-\mathrm{C}, \mathrm{mmol} / \mathrm{l}$ & $1.44 \pm 0.31$ & $1.55 \pm 0.28 \mathrm{a}$ & $1.34 \pm 0.28_{b}$ & $1.06 \pm 0.20_{c}$ & $<0.01$ \\
\hline LDL-C, mmol/l & $1.84 \pm 0.44$ & $1.81 \pm 0.42_{\mathrm{a}}$ & $1.86 \pm 0.46_{\mathrm{a}}$ & $1.94 \pm 0.46_{b}$ & $<0.01$ \\
\hline \multicolumn{6}{|l|}{ Metabolic syndrome components } \\
\hline Central obesity & $428(21.83 \%)$ & - & $341(41.74 \%)$ & $87(94.57 \%)$ & $<0.01$ \\
\hline Elevated triglycerides & $499(25.45 \%)$ & - & $416(50.92 \%)$ & $83(90.22 \%)$ & $<0.01$ \\
\hline Reduced HDL-C & $182(9.28 \%)$ & - & $122(14.93 \%)$ & $60(65.22 \%)$ & $<0.01$ \\
\hline Elevated blood pressure & $192(9.79 \%)$ & - & $141(17.26 \%)$ & $51(55.43 \%)$ & $<0.01$ \\
\hline Impaired fasting glucose & $30(1.53 \%)$ & - & $27(3.30 \%)$ & $3(3.26 \%)$ & $<0.01$ \\
\hline \multicolumn{6}{|l|}{ Perinatal measures } \\
\hline \multicolumn{6}{|l|}{ Fatal weight of pregnancy week } \\
\hline Appropriate for gestational age & $1097(68.18 \%)$ & $599(69.41 \%)$ & $444(66.37 \%)$ & $54(70.13 \%)$ & 0.04 \\
\hline Small for gestational age & $126(7.83 \%)$ & $79(9.15 \%)$ & $43(6.43 \%)$ & $4(5.19 \%)$ & \\
\hline Large for gestational age & $386(23.99 \%)$ & $185(21.44 \%)$ & $182(27.20 \%)$ & $19(24.68 \%)$ & \\
\hline \multicolumn{6}{|l|}{ Mother's education, $y$} \\
\hline$\sim 9$ & $641(33.42 \%)$ & $335(32.56 \%)$ & $283(35.42 \%)$ & $23(25.56 \%)$ & 0.04 \\
\hline
\end{tabular}


Table 1 (continued)

\begin{tabular}{|c|c|c|c|c|c|}
\hline \multirow[t]{2}{*}{ Variables } & \multirow[t]{2}{*}{ All } & \multicolumn{4}{|c|}{ Metabolic syndrome (MS) } \\
\hline & & No & $1 \sim 2$ MS Components & Yes & $P$ \\
\hline$\sim 12$ & $660(34.41 \%)$ & $363(35.28 \%)$ & $254(31.79 \%)$ & $43(47.78 \%)$ & \\
\hline$\geq 15$ & $617(32.17 \%)$ & $331(32.17 \%)$ & $262(32.79 \%)$ & 24 (26.67\%) & \\
\hline \multicolumn{6}{|l|}{13 domains of $Q o L$} \\
\hline Self-satisfy & $50.07 \pm 11.35$ & $49.81 \pm 11.32$ & $50.54 \pm 11.40$ & $48.99 \pm 11.18$ & 0.25 \\
\hline Relationship of teacher and pupil & $53.59 \pm 10.08$ & $53.50 \pm 9.97$ & $53.82 \pm 10.09$ & $52.67 \pm 11.29$ & 0.53 \\
\hline Physical feeling & $49.94 \pm 10.75$ & $49.97 \pm 10.57$ & $49.99 \pm 10.89$ & $49.25 \pm 11.61$ & 0.82 \\
\hline Companionship & $53.74 \pm 10.51$ & $53.42 \pm 10.46$ & $54.33 \pm 10.30$ & $52.24 \pm 12.49$ & 0.07 \\
\hline Parenthood & $51.27 \pm 11.36$ & $51.18 \pm 11.17$ & $51.45 \pm 11.70$ & $50.81 \pm 10.64$ & 0.82 \\
\hline Physical activity ability & $50.14 \pm 10.66$ & $50.93 \pm 10.45_{a}$ & $49.48 \pm 10.80_{a}$ & $46.89 \pm 10.84_{b}$ & $<0.01$ \\
\hline Learning ability and attitude & $51.77 \pm 10.24$ & $51.50 \pm 10.15$ & $52.26 \pm 10.16$ & $50.53 \pm 11.69$ & 0.14 \\
\hline Self-esteem & $50.34 \pm 11.06$ & $50.17 \pm 10.96$ & $50.76 \pm 11.09$ & $48.64 \pm 11.83$ & 0.17 \\
\hline Negative emotion & $47.71 \pm 11.25$ & $47.57 \pm 11.26$ & $48.05 \pm 11.09$ & $46.41 \pm 12.47$ & 0.34 \\
\hline Attitude of doing homework & $51.47 \pm 9.06$ & $51.57 \pm 8.92$ & $51.48 \pm 9.05$ & $50.25 \pm 10.77$ & 0.41 \\
\hline Activity opportunity & $54.60 \pm 9.62$ & $54.16 \pm 9.59$ & $55.26 \pm 9.64$ & $53.69 \pm 9.46$ & 0.03 \\
\hline Living convenience & $54.45 \pm 7.73$ & $54.28 \pm 7.60$ & $54.57 \pm 7.96$ & $55.18 \pm 7.18$ & 0.47 \\
\hline Other (picky-eating and surroundings) & $50.66 \pm 10.12$ & $50.04 \pm 10.24 a$ & $51.25 \pm 9.93_{a}$ & $52.49 \pm 10.03_{b}$ & $<0.01$ \\
\hline \multicolumn{6}{|l|}{ Four factors of $Q o L$} \\
\hline Psychosocial health factor & $64.79 \pm 10.37$ & $64.64 \pm 10.19$ & $65.18 \pm 10.46$ & $63.13 \pm 11.45$ & 0.46 \\
\hline Physical and mental health factor & $35.87 \pm 5.98$ & $35.89 \pm 5.87$ & $35.94 \pm 6.06$ & $35.12 \pm 6.50$ & 0.16 \\
\hline Living environment factor & $24.01 \pm 4.28$ & $24.14 \pm 4.24 a$ & $23.94 \pm 4.33_{a}$ & $23.11 \pm 4.19_{b}$ & 0.07 \\
\hline Quality of life satisfaction factor & $24.90 \pm 4.37$ & $24.75 \pm 4.40$ & $25.11 \pm 4.36$ & $24.79 \pm 4.21$ & 0.21 \\
\hline Total score of QoL & $52.61 \pm 12.37$ & $52.47 \pm 12.17 \mathrm{ab}$ & $53.06 \pm 12.56_{a}$ & $50.33 \pm 12.85_{b}$ & 0.12 \\
\hline \multicolumn{6}{|l|}{ Personality characteristics } \\
\hline Extraversion (E) & $50.30 \pm 13.04$ & $50.69 \pm 13.19 a$ & $50.13 \pm 12.86_{a}$ & $47.33 \pm 12.67_{b}$ & 0.05 \\
\hline Neuroticism (N) & $50.28 \pm 14.67$ & $50.48 \pm 14.55$ & $49.80 \pm 14.60$ & $52.31 \pm 16.59$ & 0.26 \\
\hline Psychoticism (P) & $39.86 \pm 8.96$ & $39.92 \pm 8.94$ & $39.71 \pm 8.86$ & $40.58 \pm 10.11$ & 0.66 \\
\hline Lie (L) & $53.43 \pm 8.89$ & $53.41 \pm 8.67$ & $53.41 \pm 9.09$ & $53.89 \pm 9.68$ & 0.88 \\
\hline
\end{tabular}

$\mathrm{a}, \mathrm{b}, \mathrm{c}$ : the difference among groups using "a", " $\mathrm{b}$ ", "cc labelled, different letters mean the difference existed between two groups

of adolescent MS was 4.69\% (92/1961), and the prevalence rates of MS components were 21.83\% (428/1961), 25.45\% (499/1961), 9.28\% (182/1961), 9.79\% (192/1961) and $1.53 \%$ (30/1961) for central obesity, elevated TGs, reduced HDL-C, elevated BP, and IFG, respectively. Anthropometric measures, biochemical indexes, MS components, perinatal, and SES variables, QoL score, and personality characteristics among the MS groups are shown in Table 1. Age, region, foetal weight of pregnancy week and mother's education level, which were adjusted in the multivariable analysis, showed significant differences among the three groups (no MS component, 1-2 MS components and MS) (all $P<0.05$ ).

Table 1 displays the QoL and personality trait scores of children with different MS component scores. Adolescents with MS had lower QoL domain scores for PAA, activity opportunities and other (picky eating and surroundings) than their counterparts with no or 1-2 MS components $(P<0.01, P=0.03$ and $P<0.01)$. In addition, the extraversion score of personality traits was decreased in children with MS compared with their counterparts with no MS components or 1-2 MS components $(P=0.05)$.

\section{Relationship of elevated MS components with QoL and personality scores}

The association of elevated MS components in 2019 with adolescent QoL and personality traits in 2019 is shown in Additional file 4: Table S2 (unadjusted covariates) and in Table 2 (adjusted covariates). After adjusting for covariates, an elevated domain scores of PAA (OR (95\% CI) 0.964 (0.953, 0.975), $P<0.01)$ and living environment factor scores (OR (95\% CI) 0.948 (0.921, 0.977), $P<0.01)$ were protective factors against central obesity. However, the domain score of others (picky-eating and surroundings) was positively correlated with central obesity (OR (95\% CI) 1.014 (1.001, 1.026), $P=0.03$ ), elevated BP (OR (95\% CI) $1.027(1.009,1.045), P<0.01)$ and decreased 


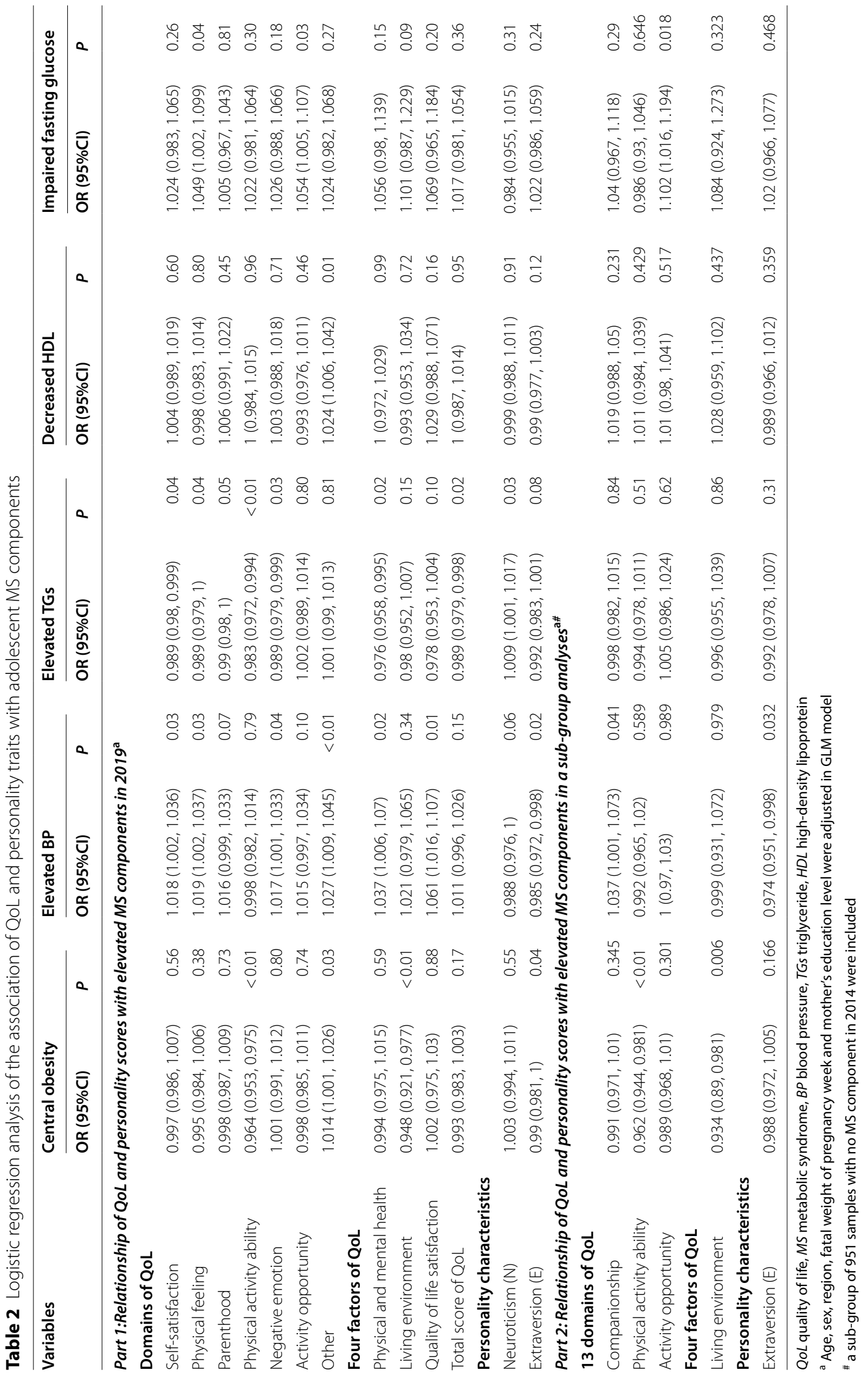


HDL (OR (95\% CI) 1.024 (1.006, 1.042), $P=0.01$ ). The QoL domain scores of self-satisfaction, physical feeling, negative emotion and factor scores of physical and mental health and quality of life satisfaction were positively correlated with elevated BP (in Table 2). In contrast, personality trait scores of extraversion (OR (95\% CI) 0.985 (0.972, 0.998), $P=0.02$ ) were negatively correlated with elevated BP. In addition, an elevated domain scores of QoL of self-satisfaction, physical feeling, parenthood, PAA, negative emotion, factor score of physical and mental health and total score of QoL were correlated with elevated TGs, while neuroticism score was positively correlated with elevated TGs (in Table 2). The domain scores of physical feeling (OR (95\% CI) 1.049 (1.002, 1.099), $P=0.04)$ and activity opportunity (OR $(95 \% \mathrm{CI}) 1.054$ $(1.005,1.107), P=0.03)$ were positively correlated with IFG.

Subgroup analyses of participants without MS components in 2014 are shown in Table 2. The results showed that PAA (OR $(95 \% \mathrm{CI}) 0.962(0.944,0.981), P<0.01)$ and living environment (OR (95\% CI) 0.934 (0.89, 0.981), $P=0.006)$ were negatively correlated with central obesity, companionship was positively correlated with elevated BP (OR (95\%CI) 1.037 (1.001, 1.073), $P=0.04)$, and activity opportunity was positively correlated with IFG (OR (95\%CI) 1.102 (1.016, 1.194), $P=0.02)$. Furthermore, extraversion personality was a protective factor against elevated BP (OR (95\% CI) 0.974 (0.951, 0.998), $P=0.03)$.

\section{Relationship of QoL and personality Taits scores with MS and MS scores in 2019}

In the multivariable logistic regression model (Table 3) (adjusted for sex, age, region, foetal weight of pregnancy week and mother's education level), the results showed that the QoL domain score of PAA was a protective factor against MS (OR (95\% CI) 0.966 (0.947, 0.986), $P<0.01)$. In addition, a high extraversion personality score was a protective factor for MS even after adjusting for sex, age, region, foetal weight of pregnancy week and mother's education level (OR (95\% CI) 0.984 (0.968, 1.000), $P=0.05)$. However, the QoL score of the "other" domain (picky eating and surroundings) was positively correlated with MS (OR (95\% CI) 1.029 (1.007, 1.052), $P=0.01)$. In the full model, the QoL domain of others (picky-eating and surroundings) was positively correlated with MS (OR (95\% CI) 1.048 (1.022, 1.075), $P<0.01)$, and the living environment factor was negatively correlated with MS (OR (95\% CI) $0.932(0.873,0.994), P<0.05)$.

After adjusting for other covariates, the GLM (Table 4) also revealed that a high PAA score was a protective factor against the MS score $(\beta=-0.008, P<0.01)$. In contrast, a high QoL score of the "other" domain was positively correlated with the MS score $(\beta=0.007$, $P<0.01$ ). In the full model, other (picky-eating and surroundings) domains were positively correlated with MS even after adjusting for covariates $(\beta=0.010, P<0.01)$, and extraversion personality was a protective factor against MS in both the total sample $(\beta=-0.006$, $P<0.01)$ and subgroup $(\beta=-0.005, P=0.03)$ analyses after adjustment for covariates.

\section{Discussion}

This study is the first prospective cohort study revealing both the longitudinal and cross-sectional correlations between QoL and MS or MS components, as well as the association between personality traits and MS or MS components over an average 12-year follow-up from birth to adolescence in urban-rural areas. We observed that an increased prevalence of MS components and MS, as well as MS score, was associated with QoL score and extraversion personality traits. The QoL domain score of PAA and extraversion personality score were independent protective factors for both MS and MS scores.

Our study revealed that centrally obese children and adolescents were mainly negatively correlated with physical QoL scores, such as PAA and factor score of living environment, and a dose-dependent relationship was found. Our result was consistent with that of other studies [31-33], showing that the obese population had lower health-related QoL (HRQoL) scores, and that weight loss would improve HRQoL [18, 34]. In our study, childhood and adolescent obesity was not significantly correlated with mental aspects of QoL scores, similar to the results of other studies [32]. In addition, this cohort study indicated that the BP level in childhood and adolescents was inversely correlated with the physical QoL score of PAA in adults [35], but it was positively correlated with mental QoL scores and total QoL scores; additionally, the mental QoL score results were not consistent with the results in adults with hypertension [36]. A study by Jing Sun et al. [37] showed that a decrease in BP would improve the physical HRQoL score in adults with hypertension, but this conclusion is controversial.

Research on the relationship between glycolipid indexes and QoL is limited. A study of adult cardiovascular disease illustrated that elevated TG levels were inversely associated with both physical and QoL scores [35]. Our study was the first to reveal that TGs were negatively related to adolescent QoL, and most of the correlations were significant in the health sub group analyses. A previous study [38] of adulthood hypertension showed that elevated HDL-C was positively correlated with the EuroQol five-dimension, three-level (EQ-5D-3L) index and EuroQol visual analogue scale (EQ VAS) score. Accordingly, we found that increased HDL-C was positively 
Table 3 Logistic regression analysis the relationship of QoL and personality traits with adolescent MS

\begin{tabular}{|c|c|c|c|c|c|c|}
\hline \multirow[t]{2}{*}{ Variables } & \multicolumn{3}{|l|}{$\mathrm{MS}^{\mathrm{a}}$} & \multicolumn{3}{|l|}{$\mathrm{MS}^{\mathrm{b}}$} \\
\hline & $\beta$ & OR $(95 \% \mathrm{Cl})$ & $P$ & $\beta$ & OR $(95 \% \mathrm{Cl})$ & $P$ \\
\hline \multicolumn{7}{|l|}{13 domains of QoL } \\
\hline Self-satisfaction & -0.006 & $0.994(0.976,1.012)$ & 0.51 & -0.006 & $0.994(0.975,1.012)$ & 0.50 \\
\hline Relationship of teacher and pupil & -0.008 & $0.992(0.972,1.013)$ & 0.45 & -0.008 & $0.992(0.971,1.013) 5$ & 0.43 \\
\hline Physical feeling & -0.006 & $0.994(0.976,1.013)$ & 0.54 & -0.007 & $0.993(0.974,1.013)$ & 0.51 \\
\hline Companionship & -0.010 & $0.990(0.971,1.009)$ & 0.31 & -0.012 & $0.988(0.969,1.007)$ & 0.23 \\
\hline Parenthood & -0.003 & $0.997(0.978,1.016)$ & 0.76 & -0.002 & $0.998(0.979,1.018)$ & 0.88 \\
\hline Physical activity ability & -0.035 & $0.965(0.946,0.985)$ & $<0.01$ & -0.034 & $0.966(0.947,0.986)$ & $<0.01$ \\
\hline Learning ability and attitude & -0.009 & $0.991(0.971,1.011)$ & 0.38 & -0.010 & $0.990(0.969,1.011)$ & 0.34 \\
\hline Self-esteem & -0.013 & $0.987(0.968,1.007)$ & 0.20 & -0.014 & $0.986(0.966,1.006)$ & 0.16 \\
\hline Negative emotion & -0.009 & $0.991(0.973,1.010)$ & 0.35 & -0.009 & $0.992(0.973,1.010)$ & 0.37 \\
\hline Attitude towards doing homework & -0.015 & $0.985(0.964,1.007)$ & 0.18 & -0.017 & $0.983(0.962,1.005)$ & 0.14 \\
\hline Activity opportunity & -0.005 & $0.995(0.973,1.017)$ & 0.65 & -0.007 & $0.993(0.971,1.015)$ & 0.53 \\
\hline Living convenience & 0.017 & $1.017(0.987,1.048)$ & 0.28 & 0.014 & $1.014(0.984,1.045)$ & 0.36 \\
\hline Other (picky-eating and surroundings) & 0.024 & $1.024(1.003,1.046)$ & 0.03 & 0.029 & $1.029(1.007,1.052)$ & 0.01 \\
\hline \multicolumn{7}{|l|}{ Four factors of QoL } \\
\hline Psychosocial & -0.014 & $0.986(0.967,1.006)$ & 0.18 & -0.012 & $0.988(0.967,1.009)$ & 0.25 \\
\hline Physical and mental health & -0.021 & $0.979(0.945,1.014)$ & 0.23 & -0.020 & $0.980(0.946,1.015)$ & 0.26 \\
\hline Living environment & -0.056 & $0.946(0.900,0.993)$ & 0.03 & -0.050 & $0.952(0.904,1.002)$ & 0.06 \\
\hline Quality of life satisfaction & 0.002 & $1.002(0.954,1.052)$ & 0.93 & 0.008 & $1.008(0.959,1.060)$ & 0.76 \\
\hline Total score of QoL & -0.014 & $0.986(0.970,1.003)$ & 0.11 & -0.015 & $0.985(0.968,1.002)$ & 0.09 \\
\hline \multicolumn{7}{|l|}{ Personality characteristics } \\
\hline Neuroticism (N) & 0.008 & $1.008(0.994,1.023)$ & 0.26 & 0.008 & $1.008(0.993,1.023)$ & 0.28 \\
\hline Psychoticism (P) & 0.008 & $1.008(0.985,1.031)$ & 0.51 & 0.008 & $1.008(0.985,1.032)$ & 0.48 \\
\hline Extraversion (E) & -0.018 & $0.982(0.967,0.997)$ & 0.02 & -0.016 & $0.984(0.968,1.000)$ & 0.05 \\
\hline \multicolumn{7}{|l|}{ Full model $^{\#}$} \\
\hline Physical activity ability & -0.012 & $0.988(0.966,1.009)$ & 0.26 & -0.014 & $0.986(0.965,1.008)$ & 0.22 \\
\hline Other (picky-eating and surroundings) & 0.045 & $1.046(1.02,1.073)$ & $<0.01$ & 0.047 & $1.048(1.022,1.075)$ & $<0.01$ \\
\hline Living environment & -0.077 & $0.926(0.87,0.986)$ & 0.02 & -0.071 & $0.932(0.873,0.994)$ & 0.03 \\
\hline Extraversion (E) & -0.012 & $0.988(0.971,1.006)$ & 0.19 & -0.011 & $0.989(0.971,1.007)$ & 0.23 \\
\hline
\end{tabular}

QoL quality of life, MS metabolic syndrome

\# The significant variables of QoL or EPQ were entered in the full model

${ }^{\text {a }}$ The crude results

${ }^{b}$ Age, sex, region, fatal weight of pregnancy week and mother's education level were adjusted in each Logistic regression model (dependent variable value: $1=$ with $\mathrm{MS}, 0$ = without any one of five MS components), and 1144 samples were included

correlated with extraversion personality traits in adolescents. Our findings revealed that the QoL score regarding learning ability and attitude has a salient effect on lipid levels in adolescents, but understanding the mechanism requires further research. In addition, FBG and HbA1c levels in type $1 \mathrm{DM}$ were negatively correlated with EQVAS (overall health status) [39], but our cohort study with an adolescent population showed that IFG in childhood was only positively correlated with the QoL score of activity opportunity. The controversial results could be explained by the different lifestyles between healthy children and adults with DM, as well as the different QoL questionnaires used between adolescents and adults.
However, a cross-sectional study showed that QoL scores were correlated with an increase in the components of MS, and the physical health domain of QoL had the most significant association [40]. In our study, we found that the PAA domain score and living environment factor score were protective factors against MS and MS scores. Previous studies have shown that health literacy training is a significant way to promote quality of life [41], suggesting that health literacy training should be provided to out in adolescents with low QoL. To our knowledge, this study is the first cohort study with a large sample size that explored the relationship of QoL with MS and MS scores in adolescents. 
Table 4 GLM analysis the association of QoL and personality traits with adolescent MS score

\begin{tabular}{|c|c|c|c|c|c|c|c|c|c|}
\hline \multirow[t]{2}{*}{ Variables } & \multicolumn{3}{|l|}{ MS* } & \multicolumn{3}{|l|}{ MS** } & \multicolumn{3}{|c|}{ MS sub-group $\#$} \\
\hline & B & StdErr & $P$ & B & StdErr & $P$ & B & StdErr & $P$ \\
\hline \multicolumn{10}{|l|}{13 domains of $Q o L$} \\
\hline Self-satisfaction & 0.001 & 0.002 & 0.73 & 0.001 & 0.002 & 0.82 & 0.002 & 0.002 & 0.33 \\
\hline Relationship of teacher and pupil & -0.002 & 0.002 & 0.38 & -0.001 & 0.002 & 0.48 & -0.002 & 0.003 & 0.50 \\
\hline Physical feeling & -0.001 & 0.002 & 0.76 & -0.001 & 0.002 & 0.65 & 0.001 & 0.002 & 0.96 \\
\hline Companionship & 0.001 & 0.002 & 0.87 & 0.001 & 0.002 & 0.94 & 0.002 & 0.002 & 0.46 \\
\hline Parenthood & 0.001 & 0.002 & 0.83 & 0.001 & 0.002 & 0.95 & -0.001 & 0.002 & 0.64 \\
\hline Physical activity ability & -0.008 & 0.002 & $<0.01$ & -0.008 & 0.002 & $<0.01$ & -0.005 & 0.002 & 0.02 \\
\hline Learning ability and attitude & 0.001 & 0.002 & 0.94 & 0.001 & 0.002 & 0.86 & -0.002 & 0.003 & 0.43 \\
\hline Self-esteem & -0.001 & 0.002 & 0.61 & -0.001 & 0.002 & 0.51 & -0.003 & 0.002 & 0.18 \\
\hline Negative emotion & 0.001 & 0.002 & 0.58 & 0.001 & 0.002 & 0.67 & 0.001 & 0.002 & 0.97 \\
\hline Attitude towards doing homework & -0.002 & 0.002 & 0.30 & -0.003 & 0.002 & 0.15 & -0.001 & 0.003 & 0.82 \\
\hline Activity opportunity & 0.003 & 0.002 & 0.11 & 0.003 & 0.002 & 0.18 & 0.001 & 0.003 & 0.69 \\
\hline Living convenience & 0.001 & 0.003 & 0.66 & 0.001 & 0.003 & 0.88 & -0.001 & 0.003 & 0.77 \\
\hline Other (picky-eating and surroundings) & 0.006 & 0.002 & $<0.01$ & 0.007 & 0.002 & $<0.01$ & 0.003 & 0.003 & 0.30 \\
\hline \multicolumn{10}{|l|}{ Four factors of QoL } \\
\hline Psychosocial & -0.002 & 0.002 & 0.38 & -0.001 & 0.002 & 0.66 & -0.001 & 0.003 & 0.57 \\
\hline Physical and mental health & -0.001 & 0.003 & 0.68 & -0.001 & 0.003 & 0.66 & 0.001 & 0.005 & 0.98 \\
\hline Living environment & -0.011 & 0.005 & 0.02 & -0.007 & 0.005 & 0.12 & -0.006 & 0.006 & 0.31 \\
\hline Quality of life satisfaction & 0.005 & 0.005 & 0.28 & 0.006 & 0.005 & 0.19 & 0.007 & 0.006 & 0.25 \\
\hline Total score of QoL & -0.001 & 0.002 & 0.46 & -0.001 & 0.002 & 0.40 & -0.001 & 0.002 & 0.50 \\
\hline \multicolumn{10}{|l|}{ Personality characteristics } \\
\hline Neuroticism (N) & 0.001 & 0.001 & 0.93 & 0.001 & 0.001 & 0.93 & 0.001 & 0.002 & 0.82 \\
\hline Psychoticism (P) & 0.001 & 0.002 & 0.99 & 0.001 & 0.002 & 0.78 & 0.002 & 0.003 & 0.57 \\
\hline Extraversion (E) & -0.004 & 0.002 & 0.01 & -0.003 & 0.002 & 0.06 & -0.005 & 0.002 & 0.03 \\
\hline \multicolumn{10}{|l|}{ Full model } \\
\hline Physical activity ability & -0.001 & 0.002 & 0.57 & -0.003 & 0.002 & 0.13 & 0.001 & 0.003 & 0.67 \\
\hline Other (picky-eating and surroundings) & 0.010 & 0.003 & $<0.01$ & 0.01 & 0.002 & $<0.01$ & - & - & - \\
\hline Living environment & -0.016 & 0.006 & 0.01 & - & - & - & - & - & - \\
\hline Extraversion (E) & -0.005 & 0.002 & $<0.01$ & -0.006 & 0.002 & $<0.01$ & -0.005 & 0.002 & 0.02 \\
\hline
\end{tabular}

*The crude results

${ }^{* *}$ Age, sex, region, fatal weight of pregnancy week and mother's education level were adjusted in Logistic regression model. QoL: quality of life, MS scores: having one metabolic syndrome components markers one score

\# Including the samples who have no MS components in 2014 ( $n=951)$

Personality traits can be associated with MS, but the conclusion remains controversial, and few studies have been conducted in adult populations; no related cohort studies have explored this relationship in adolescents. The results from Japanese adults [20] showed that the E score was positively correlated with TGs, FBG and MS components, and the $\mathrm{P}$ score was positively correlated with FBG. However, we found that the E score in adolescents was negatively correlated with MS, central obesity, and SBP and positively correlated with HDL, suggesting that the $\mathrm{E}$ score could be age dependent and have a different impact on lipid metabolism between adults and adolescents. A study by Evans [42] reported that extraverted adolescents have less cortisol activity, which is associated with FBG and FI [43]. Therefore, extraversion traits might regulate glycolipid indexes through the hypothalamicpituitary-adrenal axis pathway [42], which can impact MS by regulating the reactivity of the sympathetic nervous system (SNS) [44], and the SNS has potent effects on insulin secretion and sensitivity [45] and on lipid metabolism [46]. Adolescents with high $\mathrm{N}$ scores would be more prone to responding more strongly to a stressor, and our study did not find $\mathrm{N}$ to be correlated with MS, inconsistent with the results of a study in adults [47].

\section{Limitations}

Our study has some limitations that should be considered when interpreting the results. First, since this study was a 
bidirectional cohort study, recall bias could exist for the prenatal variables. We checked the birth certificates to verify the birth weight, stature and gestational age. Second, data on GH and gestational diabetes were collected through a questionnaire, and recall bias existed. However, we collected the same perinatal information in both 2014 and 2019 independently to reduce recall bias and nonresponse. Finally, QoL and personality traits were collected in a cross-sectional manner in 2019, making it difficult to draw conclusions regarding the causality relationships of QoL and personality traits with MS.

\section{Conclusions}

In conclusion, the prevalence of MS was elevated among adolescents. Our findings add to the scarce research evidence regarding the relationships of QoL and personality traits with MS in children and adolescents, which could facilitate the generation of policy suggestions for health management institutes. In this study, we identified the relationships of QoL and personality traits with MS and MS components from both prospective and cross-sectional aspects after adjustment for other covariates. First, the PAA score was inversely correlated with MS and MS scores, suggesting that the establishment of a comprehensive community, family and school intervention model to improve the PAA of children is essential for the control of adolescents with MS. Second, the QoL score of other domains, including picky eating and surroundings, was positively correlated with MS and MS scores, indicating that health education, including literacy training and health dietary habits, should be implemented both at home and in school to improve lifestyles. Finally, the extraversion personality trait was a protective factor against MS and MS score; therefore, primary prevention in childhood health should focus on cultivating children's extraversion personality. Further studies should explore the effect of comprehensive intervention on the control of adolescent MS by improving QoL and personality traits.

\footnotetext{
Abbreviations

MS: Metabolic syndrome; CVDs: Cardiovascular diseases; TGs: Triglycerides; HDL-C: High density lipoprotein cholesterol; SES: Socialeconomic status; QoL: Quality of life; FBG: Fasting blood-glucose; BMI: Body mass index; GH: Gestational hypertension; WC: Waist circumference; BP: Blood pressure; LDL: Low density lipoprotein cholesterol; TC: Total cholesterol; EPQ: Eysenck's Personality Questionnaire; PAA: Physical activity ability; E: Extraversion; N: Neuroticism; P: Psychoticism; L: Lie scales; IFG: Impaired fasting glucose; LGA: Large for gestational age; SGA: Small for gestational age; IOM: The Institute of Medicine; SNK: Student-Newman-Keuls; GLM: Generalized linear model; SBP: Systolic blood pressure; DBP: Diastolic blood pressure; HRQoL: Health-related QoL; SNS: Sympathetic nervous system.
}

\section{Supplementary Information}

The online version contains supplementary material available at https://doi. org/10.1186/s12955-021-01797-7.

Additional file 1: Figure S1. The hypothesized framework of this manuscript.

Additional file 2: Table S1. Comparation baseline characteristics between included and excluded participants

Additional file 3: Figure $\mathbf{S 2}$. The structure chart of quality of life Additional file 4: Table S2. Logistic regression analysis of the association of QoL and personality traits with adolescent MS components without adjusting other covariates

\section{Acknowledgements}

The authors would like to acknowledge the laboratory support of the Ministry of Education Key Laboratory of Child Development and Disorders and all of the staff members of the 6 elementary schools in the two regions.

\section{Authors' contributions}

$\mathrm{XH}$ conceived and designed the experiments; $\mathrm{PZ}$ and SQ performed the experiments; WF, GF, XT and $L J$ participated in the physical measurement; $\mathrm{XH}$ wrote the paper; and all of the authors critically reviewed and approved the final paper. The authors would like to acknowledge the laboratory support of the Ministry of Education Key Laboratory of Child Development and Disorders and all of the staff members of the 6 elementary schools in the two regions. All authors read and approved the final manuscript.

\section{Funding}

This work was supported by the Joint Medical Research Project of Chongqing Municipal Health Commission and Chongqing Science and Technology Bureau (No. 2020MSXM062), the Basic Research Project of Key Laboratory of Ministry of Education of China in 2021 (GBRP-202106), the National Key Research and Development Project of the Ministry of Science and Technology of the People's Republic of China (No.2017YFC0211705), Young Scientists Fund Program of the National Natural Science Foundation of China (No.81502826), School Funded Project of Chongqing Medical University (No.CQMUNCP0204), Young Scientists Fund Program of the Education Commission of Chongqing (No.KJQN201900443), General Program of the China Postdoctoral Science Foundation (No.2014M562289), and Chongqing Postdoctoral Research Funded Projects (No.Xm2014129). The funders had no role in the study design, the data collection and analysis, the decision to publish, or the preparation of the manuscript.

\section{Availability of data and materials}

Data are available from Xiaohua Liang (contact via xiaohualiang@hospital. cqmu.edu.cn, or liangxiaohua666@sina.com).

\section{Declarations}

Ethics approval and consent to participate

The Institutional Review Board at the Children's Hospital of Chongqing Medical University gave its approval for the study. Informed consent was provided by all of the subjects and parents/guardians. Informed consent to participate in this study was provided by all of the subjects and parents/guardians.

\section{Consent for publication}

All of the authors critically reviewed and approved the publication of the final paper.

\section{Competing interests}

The authors have no relevant conflicts of interest to disclose.

\section{Author details}

${ }^{1}$ Clinical Epidemiology and Biostatistics Department, Children's Hospital of Chongqing Medical University, Ministry of Education Key Laboratory of Child Development and Disorders, National Clinical Research Center 
for Child Health and Disorders, Key Laboratory of Pediatrics in Chongqing, China International Science and Technology Cooperation Center of Child Development and Critical Disorders, 136 2nd Street, Yuzhong District, Chongqing 400016, China. ${ }^{2}$ Disease Control and Prevention Center of Jiulongpo District, Chongqing, China. ${ }^{3}$ Medical General Ward of Children's Hospital of Chongqing Medical University, Chongqing, China. ${ }^{4}$ Plastic Surgery Department of Children's Hospital of Chongqing Medical University, Chongqing, China.

Received: 31 August 2020 Accepted: 2 June 2021

Published online: 08 June 2021

\section{References}

1. Koskinen JS, Kyto V, Juonala M, Viikari JSA, Nevalainen J, Kahonen M, Lehtimaki T, Hutri-Kahonen N, Laitinen T, Tossavainen P, et al. Childhood risk factors and carotid atherosclerotic plaque in adulthood: The Cardiovascular Risk in Young Finns Study. Atherosclerosis. 2019;293:18-25.

2. DeBoer MD, Gurka MJ, Woo JG, Morrison JA. Severity of metabolic syndrome as a predictor of cardiovascular disease between childhood and adulthood: the Princeton Lipid Research Cohort Study. J Am Coll Cardiol. 2015;66:755-7.

3. Johnson WD, Kroon JJ, Greenway FL, Bouchard C, Ryan D, Katzmarzyk PT. Prevalence of risk factors for metabolic syndrome in adolescents: National Health and Nutrition Examination Survey (NHANES), 2001-2006. Arch Pediatr Adolesc Med. 2009;163:371-7.

4. Li Y, Yang X, Zhai F, Kok FJ, Zhao W, Piao J, Zhang J, Cui Z, Ma G. Prevalence of the metabolic syndrome in Chinese adolescents. Br J Nutr. 2008;99:565-70

5. Magge SN, Goodman E, Armstrong SC, Committee On N, Section On E, Section On O. The metabolic syndrome in children and adolescents: shifting the focus to cardiometabolic risk factor clustering. Pediatrics. 2017;140(2):e20171603.

6. Fadzlina AA, Harun F, Nurul Haniza MY, AI Sadat N, Murray L, Cantwell MM, Su TT, Majid HA, Jalaludin MY. Metabolic syndrome among 13 year old adolescents: prevalence and risk factors. BMC Public Health. 2014;14(Suppl 3):S7.

7. Lowe WL Jr, Scholtens DM, Kuang A, Linder B, Lawrence JM, Lebenthal Y, McCance D, Hamilton J, Nodzenski M, Talbot O, et al. Hyperglycemia and adverse pregnancy outcome follow-up study (HAPO FUS): maternal gestational diabetes mellitus and childhood glucose metabolism. Diabetes Care. 2019;42:372-80.

8. He W, James SA, Merli MG, Zheng H. An increasing socioeconomic gap in childhood overweight and obesity in China. Am J Public Health. 2014;104:e14-22.

9. Iglayreger HB, Peterson MD, Liu D, Parker CA, Woolford SJ, Sallinen Gafka BJ, Hassan F, Gordon PM. Sleep duration predicts cardiometabolic risk in obese adolescents. J Pediatr. 2014;164:1085-1090 e1081.

10. Saboya PP, Bodanese LC, Zimmermann PR, Gustavo AD, Assumpcao CM Londero F. Metabolic syndrome and quality of life: a systematic review. Rev Lat Am Enfermagem. 2016;24:e2848.

11. Amiri P, Deihim T, Taherian R, Karimi M, Gharibzadeh S, Asghari-Jafarabadi $M$, Shiva N, Azizi F. Factors affecting gender differences in the association between health-related quality of life and metabolic syndrome components: Tehran Lipid and Glucose Study. PLoS ONE. 2015;10(12):e0143167.

12. Vetter ML, Wadden TA, Lavenberg J, Moore RH, Volger S, Perez JL, Sarwer DB, Tsai AG. Relation of health-related quality of life to metabolic syndrome, obesity, depression and comorbid illnesses. Int J Obes (Lond). 2011;35:1087-94.

13. Lidfeldt J, Nyberg P, Nerbrand C, Samsioe G, Schersten B, Agardh CD. Socio-demographic and psychosocial factors are associated with features of the metabolic syndrome. The Women's Health in the Lund Area (WHILA) study. Diabetes Obes Metab. 2003;5:106-12.

14. Sarrafzadegan N, Gharipour M, Ramezani MA, Rabiei K, Zolfaghar B, Tavassoli AA, Boshtam M, Zarfeshani S, Khosravi A, Yousefi A. Metabolic syndrome and health-related quality of life in Iranian population. J Res Med Sci. 2011;16:254-61.

15. Taylor TR, Dash C, Sheppard V, Makambi K, Ma X, Adams-Campbell LL. The effect of a randomized controlled physical activity trial on health related quality of life in metabolically unhealthy African-American women: FIERCE STUDY. Contemp Clin Trials. 2018;67:121-8.

16. Wu XY, Han LH, Zhang JH, Luo S, Hu JW, Sun K. The influence of physical activity, sedentary behavior on health-related quality of life among the general population of children and adolescents: a systematic review. PLOS ONE. 2017;12:e0187668.

17. Wu XY, Zhuang LH, Li W, Guo HW, Zhang JH, Zhao YK, Hu JW, Gao QQ Luo S, Ohinmaa A, Veugelers PJ. The influence of diet quality and dietary behavior on health-related quality of life in the general population of children and adolescents: a systematic review and meta-analysis. Qual Life Res. 2019;28:1989-2015.

18. Mollerup PM, Nielsen TRH, Bojsoe C, Kloppenborg JT, Baker JL, Holm JC. Quality of life improves in children and adolescents during a community-based overweight and obesity treatment. Qual Life Res. 2017;26:1597-608.

19. Sagar R, Gupta T. Psychological aspects of obesity in children and adolescents. Indian J Pediatr. 2018;85:554-9.

20. Ohseto H, Ishikuro M, Kikuya M, Obara T, Igarashi Y, Takahashi S, Kikuchi D, Shigihara M, Yamanaka C, Miyashita M, et al. Relationships among personality traits, metabolic syndrome, and metabolic syndrome scores: the Kakegawa cohort study. J Psychosom Res. 2018;107:20-5.

21. Damian RI, Spengler M, Sutu A, Roberts BW. Sixteen going on sixty-six: a longitudinal study of personality stability and change across 50 years. J Pers Soc Psychol. 2019;117:674-95.

22. Liang XH, Xiao L, Luo YT, Xu JP. Prevalence and risk factors of childhood hypertension from birth through childhood: a retrospective cohort study. J Hum Hypertens. 2020;34:151-64.

23. Hanrong WUPL. Heng MENG norn, reliability and validity of children and adolescents' QOL scale. Chin J School Health. 2006;27:18-21.

24. Yang Y. Rating scales for children's developmental behavior and mental health. Beijing: People's Medical Publishing Co. Ltd; 2016.

25. Wang Q, Yin J, Xu L, Cheng H, Zhao X, Xiang H, Lam HS, Mi J, Li M. Prevalence of metabolic syndrome in a cohort of Chinese schoolchildren: comparison of two definitions and assessment of adipokines as components by factor analysis. BMC Public Health. 2013;13:249.

26. Ma G, Ji C, Ma J, Mi J, Ry S, Xiong F, Yan W, Hu X, Li Y, Du S, Fang H, Jiang J. Waist circumference reference values for screening cardiovascular risk factors in Chinese children and adolescents aged $7-18$ years. Chin J Epidemiol. 2010;31:609-15.

27. Fan $\mathrm{H}, \mathrm{Yan} \mathrm{Y}, \mathrm{Mi}$ J, on behalf of the Chinese Child Blood Pressure References Collaborative Group. Updating blood pressure references for Chinese children aged 3-17 years. Chin J Hypertens. 2017;25:428-435.

28. Mikolajczyk RT, Zhang J, Betran AP, Souza JP, Mori R, Gulmezoglu AM, Merialdi M. A global reference for fetal-weight and birthweight percentiles. Lancet. 2011;377:1855-61.

29. Hu L, Huang X, You C, Li J, Hong K, Li P, Wu Y, Wu Q, Wang Z, Gao R, et al. Prevalence of overweight, obesity, abdominal obesity and obesity-related risk factors in southern China. PLoS ONE. 2017;12:e0183934.

30. Johnson J, Clifton RG, Roberts JM, Myatt L, Hauth JC, Spong CY, Varner MW, Wapner RJ, Thorp JM Jr, Mercer BM, et al. Pregnancy outcomes with weight gain above or below the 2009 Institute of Medicine guidelines. Obstet Gynecol. 2013;121:969-75.

31. Kesztyus D, Wirt T, Kobel S, Schreiber A, Kettner S, Dreyhaupt J, Kilian R, Steinacker JM, Komm mit in das gesunde Boot - Grundschule" - Research G. Is central obesity associated with poorer health and health-related quality of life in primary school children? Cross-sectional results from the Baden-Wurttemberg Study. BMC Public Health. 2013;13:260.

32. Kolotkin RL, Andersen JR. A systematic review of reviews: exploring the relationship between obesity, weight loss and health-related quality of life. Clin Obes. 2017;7:273-89.

33. Kesztyus D, Schreiber A, Kobel S, Wartha O, Kesztyus T, Kilian R, Steinacker JM, Study group "Join the Healthy Boat - Primary S. Illness and determinants of health-related quality of life in a cross-sectional sample of schoolchildren in different weight categories. Ger Med Sci. 2014; 12:Doc04.

34. Poll FA, Miraglia F, D'Avila HF, Reuter CP, Mello ED. Impact of intervention on nutritional status, consumption of processed foods, and quality of life of adolescents with excess weight. J Pediatr (Rio J). 2020;96(5):621-9.

35. Uchmanowicz I, Loboz-Grudzien K, Jankowska-Polanska B, Sokalski L. Influence of diabetes on health-related quality of life results in patients 
with acute coronary syndrome treated with coronary angioplasty. Acta Diabetol. 2013;50:217-25.

36. Adedapo AD, Akunne OO, Adedokun BO. Comparative assessment of determinants of health-related quality of life in hypertensive patients and normal population in south-west Nigeria. Int J Clin Pharmacol Ther. 2015;53:265-71.

37. Sun J, Buys N. Community-based mind-body meditative Tai Chi program and its effects on improvement of blood pressure, weight, renal function, serum lipoprotein, and quality of life in Chinese Adults with Hypertens. Am J Cardiol. 2015;116:1076-81.

38. Yan R, Gu HQ, Wang W, Ma L, Li W, Group CR. Health-related quality of life in blood pressure control and blood lipid-lowering therapies: results from the CHIEF randomized controlled trial. Hypertens Res. 2019:42:1561-71.

39. Braga de Souza AC, Felicio JS, Koury CC, Neto JF, Mileo KB, Santos FM, Negrato CA, Motta AR, Silva DD, Arbage TP, et al. Health-related quality of life in people with type 1 Diabetes Mellitus: data from the Brazilian Type 1 Diabetes Study Group. Health Qual Life Outcomes. 2015;13:204.

40. Amiri P, Deihim T, Taherian R, Karimi M, Gharibzadeh S, Asghari-Jafarabadi $M$, Shiva N, Azizi F. Factors affecting gender differences in the association between health-related quality of life and metabolic syndrome components: Tehran Lipid and Glucose Study. PLoS ONE. 2015;10:e0143167.

41. Khandehroo M, Tavakoly Sany SB, Oakley D, Peyman N. Health literacy intervention and quality of life in menopausal women: a randomized controlled trial. Int J Health Promot Educ. 2020;5:1-14.

42. Evans BE, Stam J, Huizink AC, Willemen AM, Westenberg PM, Branje $\mathrm{S}$, Meeus W, Koot HM, van Lier PAC. Neuroticism and extraversion in relation to physiological stress reactivity during adolescence. Biol Psychol. 2016;117:67-79.

43. Park SB, Blumenthal JA, Lee SY, Georgiades A. Association of cortisol and the metabolic syndrome in Korean men and women. J Korean Med Sci. 2011;26:914-8.

44. Koolhaas JM, de Boer SF, Buwalda B, van Reenen K. Individual variation in coping with stress: a multidimensional approach of ultimate and proximate mechanisms. Brain Behav Evol. 2007;70:218-26.

45. Morton GJ, Muta K, Kaiyala KJ, Rojas JM, Scarlett JM, Matsen ME, Nelson JT, Acharya NK, Piccinini F, Stefanovski D, et al. Evidence that the sympathetic nervous system elicits rapid, coordinated, and reciprocal adjustments of insulin secretion and insulin sensitivity during cold exposure. Diabetes. 2017;66:823-34.

46. Geerling JJ, Boon MR, Kooijman S, Parlevliet ET, Havekes LM, Romijn JA, Meurs IM, Rensen PC. Sympathetic nervous system control of triglyceride metabolism: novel concepts derived from recent studies. J Lipid Res. 2014:55:180-9.

47. van Doornen LJ, Orlebeke KF. Stress, personality and serum-cholesterol level. J Hum Stress. 1982;8:24-9.

\section{Publisher's Note}

Springer Nature remains neutral with regard to jurisdictional claims in published maps and institutional affiliations.
Ready to submit your research? Choose BMC and benefit from:

- fast, convenient online submission

- thorough peer review by experienced researchers in your field

- rapid publication on acceptance

- support for research data, including large and complex data types

- gold Open Access which fosters wider collaboration and increased citations

- maximum visibility for your research: over 100M website views per year

At BMC, research is always in progress.

Learn more biomedcentral.com/submissions 